\title{
Comparison of functional outcome and quality of life following treatment of unstable intertrochanteric fractures with intramedullary nailing and dynamic hip screw
}

\author{
Reddy A.K. ${ }^{1}$, Reddy R.C.S. ${ }^{2}$ \\ ${ }^{1}$ Dr. Anil Kumar Reddy, Assistant Professor, Department of Orthopedics, Rajiv Gandhi Institute of Medical Sciences, \\ Kadapa, Andhra Pradesh, India, ${ }^{2}$ Dr. R. Chandra Sekhar Reddy, Assistant Professor, Department of Orthopedics, Fathima \\ Institute of Medical Sciences, Kadapa, Andhra Pradesh, India.
}

Corresponding Author: Dr. R. Chandra Sekhar Reddy, Assistant Professor, Department of Orthopedics, Fathima Institute of Medical Sciences, Kadapa, Andhra Pradesh, India. E-mail: kadapadoctors@gmail.com

\begin{abstract}
Background: The dynamic hip screw is considered as the standard treatment for Intertrochanteric fracture. The procedure per se requires long term hospitalization and other complications. Researchers have proposed intramedullary nailing as an alternate procedure. The present study aims to compare the radiological and functional outcome following treatment of unstable intertrochanteric fractures with intramedullary nailing and dynamic hip screw and to compare Health-related quality of life between the two treatment methods. Methods: A prospective observational study was conducted during March 2014 to July 2016. Patients presenting with unstable Intertrochanteric fractures were recruited prospectively. The study was conducted in the department of orthopedics, Rajiv Gandhi Institute of medical sciences, Kadapa, which is a tertiary care teaching hospital. The participants were divided into two groups. Group, I constitute 25 patients who underwent DHS and Group II had 23 patients who underwent IHN. The mean age of Group DHS participants were $71.4 \pm 5.86$ years, and group IMN participants were $69.7 \pm 6.13$ years. The mean intraoperative bleeding of subjects in DHS group was325.24 \pm 67.32 (in ml) and it was $325.24 \pm 67.32$ (in ml) in IMN group. Results: The mean time taken for the radiological union of subjects in DHS group was22.3 \pm 1.73 weeks, and it was 20.6 \pm 2.13 weeks in IMN group. Conclusions: The treatment of unstable Intertrochanteric fracture can be done in geriatric patients through surgical intervention without much morbidity and mortality. The postoperative quality of life returns to near normal at the end of one year period.
\end{abstract}

Keywords: Intertrochanteric fracture, Quality of life, Intramedullary nailing, Dynamic Hip Screw

\section{Introduction}

Intertrochanteric fractures of the femur are the most common fractures occurring in geriatric age group patients. It is associated with high morbidity and mortality. The interventional management requires long term hospitalization and bed rest causing complications of its own. The most important treatment goal is early rehabilitation achieved by stable reduction and firm internal fixation [1]. The surgical treatment for intertrochanteric fracture of the femur is done using orthopedic implants. The choice of implant should be based on the pattern of fracture and the patient's condition. Nevertheless, it also depends on the choice of the operating surgeon, surgeon's expertise and

Manuscript Received: $30^{\text {th }}$ July 2019

Reviewed: $10^{\text {th }}$ August 2019

Author Corrected: $18^{\text {th }}$ August 2019

Accepted for Publication: $22^{\text {nd }}$ August 2019 preference. For a long time, dynamic hip screws (DHS) were the main treatment option, but intramedullary nailing has gained popularity in recent times [2, 3]. Many researchers have reported on theoutcomes of DHS and proximal femoral nails in the treatment of unstable trochanteric fractures [4] however, there have been few studies regarding the treatment outcomes for stable trochanteric fractures, as such fractures can be easily overlooked.

The surgical treatment remains uncontroversial. But, the condition in elderly patients should be taken into account. Comorbidities associated with ageing often increases the risk of morbidity and mortality associated with fractures in the geriatric population. The postoperative outcome considered in previous studies is 


\section{Original Research Article}

merely radiological union [5]. It is equally important to consider the patients perspective on the surgical outcome like reduction in pain, increased mobility, self care etc. the quality of life lead after the surgical intervention is essential, and research into this aspect is lacking. Hence, this study was planned with an aim to fulfil the lacunae.

\section{Objectives}

- To compare the radiological and functional outcome following treatment of unstable intertrochanteric fractures with intramedullary nailing and dynamic hip screw

- To compare Health-related quality of life between the two treatment methods

\section{Methods}

Study setting:Department of orthopedics, Rajiv Gandhi Institute of medical sciences, Kadapa, which is a tertiary care teaching hospital.

Type of study: A prospective observational study Sample size \&Sampling method:

Inclusion \& exclusion criteria: The study population included people above 60 years, presenting with an unstable intertrochanteric fracture of the femur, which included displaced greater trochanteric fractures, fractures, which could not be reduced prior to internal fixation, fractures involving large fragments in posteromedial aspect, fractures with the baso-cervical pattern. People below 60 years and above 80 years were excluded from the study.

The other exclusion criteria considered were people with a prior history of fracture involving the hip, prior hip surgery of the involved side. People with multiple fractures and people with serious co-morbidities were also excluded from the study.

Sample size: The primary outcome considered for sample size calculation included was time taken for the radiological union.As per a study published by Cho HM et al. [6], the expected time taken for the union in weeks was 21.5 weeks in DHS group.

Assuming clinically significant difference as one week, with a common standard deviation of 1 , the required sample size was calculated assuming $90 \%$ power and $5 \%$ two-sided alpha error. The required sample size was as per the above parameters was 23 subjects in each group. To account for loss to follow up of about $10 \%$, another three subjects were added, and a total of 26 subjects were included in each group. The study sample was selected by convenient sampling

Surgical procedure: The surgical procedure was decided as per the standardized institutional protocol and also by the preference of the patient. Hence no random allocation was done in this study. The study was approved by the institutional ethical committee. Informed written consent was obtained from all the study subjects and confidentiality of the study participants was maintained.

Both the surgical procedures were performed as per the institutional protocol. All the study participants were followed up at weekly interval till the end of the first month and at monthly intervals till six months postoperatively thereafter. The key outcome parameters assessed were time taken for the radiological union, Time is taken for full weight bearing, post-operative complications. Functional outcome and quality of life at 3rd and 6th month follow up period.

The data was collected using a structured study proforma. The functional outcome was assessed by the Harris Hip Score (HHS), and the quality of life was assessed by health-related quality of life (HrQOL) questionnaire.

Ethical issues: The study was approved by Institutional Human Ethical committee. Informed written consent was obtained from all the study participants. Confidentiality of the study participants was maintained.

Statistical Methods: Clinical, radiological and functional outcome (Harris hip score) were considered as primary outcome variables.

Age, gender, comorbidities were considered as other study relevant variables.

Study group (DHS Vs IMN) was considered as Primary explanatory variable. For normally distributed Quantitative parameters the mean values were compared between study groups using Independent sample t-test (2 groups).

Categorical outcomes were compared between study groups using Chi square test. $\mathrm{P}$ value $<0.05$ was considered statistically significant. IBM SPSS version 22 was used for statistical analysis. 


\section{Original Research Article}

\section{Result}

The mean age of subjects in DHSgroup was $71.4 \pm 5.86$ years and it was $69.7 \pm 6.13$ years in IMN group. The difference in the mean age between the two groups was statistically not significant (P Value 0.331$)$. In the DHS group, 19 (76\%) participants were male, and $6(24 \%)$ participants were female. In the IMN group, 18 (78.26\%) participants were male, and $5(21.74 \%)$ participants were female. The difference in the proportion of gender between study groups was statistically not significant (P value 0.853 ).

The difference in diabetes mellitus between the study groups is found to be insignificant with a $P$ value of 0.386 . The difference in hypertension between the study groups is found to be insignificant with a P-value of 0.459 . The difference in the history of CAD between the study groups is found to be insignificant with a $\mathrm{P}$ value of 0.994 . The difference in the history of pulmonary disease between the study groups is found to be insignificant with a P- value of 0.853 . The mean BMI of subjects in the DHS group was $25.3 \pm 4.53$ and it was $24.95 \pm 5.13$ in IMN group. The difference in the mean BMI between the two groups was statistically not significant (P Value 0.802). The mean operative times of subjects in DHSgroup was $25.3 \pm 4.53$ minutes and it was $24.95 \pm 5.13$ minutes in IMN group.

The difference in the mean operative times between the two groups was statistically significant (P Value 0.001 ). The mean intraoperative bleeding of subjects in the DHSgroup was $325.24 \pm 67.32$ (in ml), and it was $325.24 \pm 67.32$ (in $\mathrm{ml}$ ) in IMN group. The difference in the mean intraoperative bleeding between the two groups was statistically not significant (P Value 0.100). The mean total duration of follow up (in months) of subjects in the DHSgroup was $13.57 \pm 1.82$ months, and it was $14.21 \pm 1.59$ months in IMN group. The difference in the mean total duration of follow up (in months) between the two groups was statistically not significant (P Value 0.202) (Table 1)

Table-1: Comparison of baseline characteristics of the two study groups

\begin{tabular}{|c|c|c|c|}
\hline \multirow{2}{*}{ Parameter } & \multicolumn{2}{|c|}{ Study group } & \multirow{2}{*}{$P$ value } \\
\hline & DHS (N=25) & IMN (N=23) & \\
\hline Age $($ Mean \pm SD) & $71.4 \pm 5.86$ & $69.7 \pm 6.13$ & 0.331 \\
\hline \multicolumn{4}{|l|}{ Gender } \\
\hline Male & $19(76 \%)$ & $18(78.26 \%)$ & \multirow{2}{*}{0.853} \\
\hline Female & $6(24 \%)$ & $5(21.74 \%)$ & \\
\hline BMI & $25.3 \pm 4.53$ & $24.95 \pm 5.13$ & 0.802 \\
\hline \multicolumn{4}{|l|}{ CO-Morbidities } \\
\hline Diabetes mellitus N (\%) & $11(44 \%)$ & $13(56.52 \%)$ & 0.386 \\
\hline Hypertension N (\%) & $16(64)$ & $17(73.91 \%)$ & 0.459 \\
\hline History of CAD N (\%) & $9(36 \%)$ & $8(34.78 \%)$ & 0.994 \\
\hline History of pulmonary disease N (\%) & $6(24 \%)$ & $5(21.74 \%)$ & 0.853 \\
\hline Operative times (In minutes) $($ mean \pm SD) & $89.78 \pm 13.57$ & $69.13 \pm 11.97$ & 0.001 \\
\hline Intraoperative bleeding (in ml)(mean \pm SD) & $325.24 \pm 67.32$ & $325.24 \pm 67.32$ & 1.000 \\
\hline The total duration of follow up (in months) (mean \pm SD) & $13.57 \pm 1.82$ & $14.21 \pm 1.59$ & 0.202 \\
\hline
\end{tabular}

The mean time taken for the radiological union of subjects in the DHSgroup was $22.3 \pm 1.73$ weeks, and it was $20.6 \pm 2.13$ weeks in IMN group. The difference inthe meantime taken for radiological union between the two groups was statistically significant ( $\mathrm{P}$ Value 0.003). The mean time is taken for weight bearing of subjects in the DHS group was $6.3 \pm 0.56$ weeks, and it was $5.8 \pm 0.47$ weeks in IMN group. The difference inthe meantime taken for weight bearing between the two groups was statistically significant (P Value 0.001). The mean shortening of the limb of subjects in DHS group was $5.38 \pm 1.15 \mathrm{~mm}$ and it was $4.09 \pm 0.97 \mathrm{~mm}$ in IMN group. The difference inthe mean shortening of the limb between the two groups was statistically significant (P Value 0.001). The different functional outcome (Harris hip score) between the study groups is found to be insignificant with a P- value of 0.858 . (Table 2) 
Original Research Article

Table-2: Comparison of clinical, radiological and functional outcome parameters between two study groups

\begin{tabular}{|l|c|c|c|}
\hline \multirow{2}{*}{ Parameter } & \multicolumn{2}{|c|}{ Study group } & \multirow{2}{*}{ P value } \\
\cline { 2 - 3 } & DHS (N=25) & IMN (N=23) & \\
\hline Time is taken for radiological union(week)(mean \pm SD) & $22.3 \pm 1.73$ & $20.6 \pm 2.13$ & 0.003 \\
\hline Time is taken for weight bearing (weeks) & $6.3 \pm 0.56$ & $5.8 \pm 0.47$ & 0.001 \\
\hline Shortening of the limb (in mm) & $5.38 \pm 1.15$ & $4.09 \pm 0.97$ & 0.001 \\
\hline Functional outcome (Harris Hip score) & $4(16 \%)$ & $3(13.04 \%)$ & \\
\hline Excellent & $11(44 \%)$ & $13(56.52 \%)$ & \multirow{2}{*}{0.858} \\
\hline Good & $7(28 \%)$ & $5(21.74 \%)$ & \\
\hline Fair & $3(12 \%)$ & $2(8.69 \%)$ & \\
\hline Poor & &
\end{tabular}

Table-3: Comparison of quality of life at 12 month follow up period between the two groups

\begin{tabular}{|c|c|c|c|}
\hline \multirow{2}{*}{ Parameter } & \multicolumn{2}{|c|}{ Study group } & \multirow{2}{*}{ P value } \\
\hline & DHS $(\mathrm{N}=25)$ & IMN (N=23) & \\
\hline \multicolumn{4}{|l|}{ Mobility } \\
\hline Level-I & $4(16 \%)$ & $2(8.69 \%)$ & \multirow{3}{*}{0.746} \\
\hline Level-II & $19(76 \%)$ & $19(82.60 \%)$ & \\
\hline Level-III & $2(8 \%)$ & $2(8.69 \%)$ & \\
\hline \multicolumn{4}{|l|}{ Self-care } \\
\hline Level-I & $15(60 \%)$ & $12(52.17 \%)$ & \multirow{3}{*}{0.704} \\
\hline Level-II & $7(28 \%)$ & $9(39.13 \%)$ & \\
\hline Level-III & $3(12 \%)$ & $2(8.69 \%)$ & \\
\hline \multicolumn{4}{|c|}{ Usual activities } \\
\hline Level-I & $9(36 \%)$ & $7(30.43 \%)$ & \multirow{3}{*}{0.658} \\
\hline Level-II & $11(44 \%)$ & $13(56.52 \%)$ & \\
\hline Level-III & $5(20 \%)$ & $2(8.69 \%)$ & \\
\hline \multicolumn{4}{|c|}{ Pain/discomfort } \\
\hline Level-I & $7(28 \%)$ & $5(27.73 \%)$ & \multirow{3}{*}{0.686} \\
\hline Level-II & $13(52 \%)$ & $11(47.82 \%)$ & \\
\hline Level-III & $5(20 \%)$ & $7(30.43 \%)$ & \\
\hline \multicolumn{4}{|c|}{ Anxiety/Depression } \\
\hline Level-I & $13(52 \%)$ & $10(43.47 \%)$ & \multirow{3}{*}{0.701} \\
\hline Level-II & $9(36 \%)$ & $11(47.82 \%)$ & \\
\hline Level-III & $3(12 \%)$ & $2(8.69 \%)$ & \\
\hline
\end{tabular}

Among the DHS group with levels of mobility, 4 (16\%) participants had level I, 19 (76\%) participants had level II and 2 (8\%) participants had level III. Among IMN group with levels of mobility, 2 (8.69\%) participants had level I, 19 (82.60\%) participants had level II and $2(8.69 \%)$ participants had level III. The difference in the proportion of levels of mobilitybetween the study groups was statistically not significant (P value 0.746 ). Among the DHS group with levels of self-care, 15 (60\%) participants had level I, 7 (28\%) participants had level II and 3 (12\%) participants had level III. Among IMN group with levels of self-care, 12 (52.17\%) participants had level I, 9 (39.13\%) participants had level II and 


\section{Original Research Article}

$2(8.69 \%)$ participants had level III. The difference in the proportion of levels of self-carebetween the study groups was statistically not significant (P value 0.704). Among DHS group with levels of usual activities, 9 (36\%) participants had level I, 11 (44\%) participants had level II and 5 (20\%) participants had level III. Among IMN group with levels of usual activities, 7 (30.43\%) participants had level I, 13 (56.52\%) participants had level II and 2 (8.69\%) participants had level III. The difference in the proportion of levels of usual activities between the study groups was statistically not significant (P value 0.658). Among DHS group with levels of pain/discomfort, 7 (28\%) participants had level I, 13 (52\%) participants had level II and 5 (20\%) participants had level III.

Among IMN group with levels of pain/discomfort, 5 (27.73\%) participants had level I, 11 (47.82\%) participants had level II and $7(30.43 \%)$ participants had level III. The difference in the proportion of levels of pain/discomfort between the study groups was statistically not significant (P value 0.686). Among DHS group with levels of anxiety/depression, 13 (52\%) participants had level I, 9 (36\%) participants had level II and 3 (12\%) participants had level III. Among IMN group with levels of anxiety/depression, 10 (43.47\%) participants had level I, 11 (47.82\%) participants had level II and 2 (8.69\%) participants had level III. The difference in the proportion of levels of anxiety/depression between the study groups was statistically not significant (P value 0.701$)$.

\section{Discussion}

Postoperative reduction and quality of life in intertrochanteric fracture correction depend on the type of fracture, an age of the patient, comorbidities, type of fixation used etc [7]. No prospective study has been done in this area regarding the postoperative quality of life of patients with Intertrochanteric fractures. This study can act as a first of its kind and set a hypothesis that can be improved in a future array of studies. This study followed up all the participants, and there was no loss to follow up, giving the study its strength.

The mean total duration of follow up (in months) of subjects in the DHS group was $13.57 \pm 1.82$ months, and it was $14.21 \pm 1.59$ months in IMN group. During the follow-up duration, the participants were enquired about the perceived quality of life. The radiological feature suggesting treatment success was radiological bone union. The mean time taken for the radiological union of subjects in the DHS group was $22.3 \pm 1.73$ weeks, and it was $20.6 \pm 2.13$ weeks in IMN group. The difference in the meantime taken for radiological union between the two groups was statistically significant.

The difference in the meantime taken for weight bearing between the two groups was statistically significant. This suggests that mobility after one year postoperative follow up was satisfactory.

A male preponderance was present in the study population. A most common cause for fracture is fall and education elderly patients and their caregivers regarding the precautions to prevent fall is vital. A large majority of the study participants suffered from other comorbidities. Mean shortening of a limb of approximately $5.3 \mathrm{~mm}$ and $4.0 \mathrm{~mm}$ was seen in group DHS and group IMN respectively.
The majority, i.e., $44 \%$ of the participants had good functional outcome based on Harris hip score in group DHS. $56.5 \%$ in group IMN had a good functional outcome at the end of follow up period.

Comparison of the quality of life after 12 months follow up period after the surgical intervention in both groups was done. It showed that $76 \%$ in group DHS and $86 \%$ in group IMN achieved level II mobility. $60 \%$ in group DHS and 52\% in group IMN achieved selfcare thereby reducing the burden on the caregivers. $44 \%$ of the participants who underwent DHS were able to do their daily activities (levelII) at the end of one year and $56.2 \%$ in IMN group. Level I anxiety and depression were seen in $52 \%$ in DHS and $43 \%$ in IMN group.

\section{Conclusion}

Time taken for radiological union was found to be slightly longer with DHS than Intra medullary nailing.

Surgical treatment by DHS had resulted in comparable functional outcome and quality of life with IMN in patients with hip fracture.Considering the lower cost and wider availability DHS may be more suitable in resource poor settings like India.

The risks, benefits and cost of the treatment shall be clearly explained to the patients to aid them in making informed decision regarding the surgery.

\section{What the study adds to the existing knowledge?}

The study had proved that treatment with DHS results in comparable functional outcome and quality of life with Intra Medually Nailing (IMN). 


\section{Original Research Article}

\section{Author's contribution}

Dr. Anil Kumar Reddy: Conceptualized the study, prepared the study protocol, participated in data collection, entry, preparation and editing of all drafts.

Dr. R. Chandra Sekhar Reddy: Review of literature and preparation of all the drafts along with contributing to the data. He had also edited and approved the final draft.

Acknowledgements:Authors would like to acknowledge the technical support in data entry, analysis and manuscript editing by "Evidence Research Associates."

\section{Declarations}

Conflict of interest: None declared. Funding: Nil, Permission from IRB: Yes

Ethical approval: Institutional ethical committee

\section{References}

1. Siegmeth A, Gurusamy K, Parker M. Delay to surgery prolongs hospital stay in patients with fractures of the proximal femur. J Bone Joint Surg Br. 2005; 87 (8):1123-1126. doi: https://doi.org/10.1302/0301-620X. 87B8. 16357

2. Rao JP, Banzon MT, Weiss AB, Rayhack J. Treatment of unstable intertrochanteric fractures with anatomic reduction and compression hip screw fixation. Clin Orthop Relat Res. 1983(175):65-71.
3. Hardy DC, Descamps P-Y, Krallis P, Fabeck L, Smets P, Bertens CL, et al. Use of an intramedullary hip-screw compared with a compression hip-screw with a plate for intertrochanteric femoral fractures. A prospective, randomized study of one hundred patients. JBJS. 1998;80(5):618-630.

4. Kumar R, Singh RN, Singh BN. Comparative prospective study of proximal femoral nail and dynamic hip screw in treatment of intertrochanteric fracture femur. J Clin Orthop Trauma. 2012; 3(1): 28-36. doi: 10. 1016/j.jcot.2011.12.001

5. Parker MJ, Handoll HH. Gamma and other cephalocondylic intramedullary nails versus extramedullary implants for extracapsular hip fractures in adults. Cochrane Database Syst Rev. 2010(Cochrane Database Syst Rev). 2010;(9):CD000093. doi: 10.1002/ 14651858.CD000093.pub5.

6. Cho HM, Lee K. Clinical and functional outcomes of treatment for type al intertrochanteric femoral fracture in elderly patients: comparison of dynamic hip screw and proximal femoral nail antirotation. Hip \& pelvis. 2016;28(4):232-242. doi: 10.5371/hp.2016.28.4.232.

7. Lorich DG, Geller DS, Nielson JH. Osteoporotic pertrochanteric hip fractures: management and current controversies. Instr Course Lect. 2004;53(2):441-454.

\section{How to cite this article?}

Reddy A.K, Reddy R.C.S. Comparison of functional outcome and quality of life following treatment of unstable intertrochanteric fractures with intramedullary nailing and dynamic hip screw. Surgical Update: Int J surg Orthopedics. 2019;5(4):253-258.doi:10.17511/ijoso.2019.i04.04 